\title{
Trauma: cervical spine
}

\author{
Timothy N. Booth
}

(C) Springer-Verlag 2011

Cervical spine injuries are relatively rare in the pediatric population; however, the consequences of missing a cervical spine injury are devastating. The routine use of radiography and multidetector CT has not been shown to be cost-effective or demonstrate improved outcomes in these patients. A carefully designed clinical protocol that includes defined parameters for reliably differentiating between patients who need imaging and those who do not is essential in the evaluation of children with cervical spinal trauma. The two largest studies evaluating cost and radiation exposure are the National Emergency X-Radiography Utilization Study (NEXUS) and the Canadian cervical spine rule (CCR). In the NEXUS study criteria for imaging included midline tenderness, altered level of alertness, intoxication, focal neurological deficit and distracting painful injury. The NEXUS criteria have been studied and have been proved reliable to separate patients into low- and high-risk groups. Additional criteria have been added by some investigators and include high-risk mechanism of injury, transient neurological symptoms, physical signs of trauma, head or face trauma and an inconsolable child. The low-risk child does not require an imaging work-up and, unlike adults, imaging of the high-risk child should begin with radiographs because of the risk of radiation exposure.

Disclaimer Dr. Booth has no financial interests, investigational or offlabel uses to disclose.

T. N. Booth $(\bowtie)$

Children's Medical Center,

Dallas, TX 75235, USA

e-mail: tim.booth@childrens.com

T. N. Booth

Department of Radiology, University of Texas

Southwestern Medical Center,

Dallas, TX 75235, USA
The initial evaluation of the pediatric cervical spine is with cervical radiographs. The level of injury is commonly higher (C1-3) in children younger than 8 years because of anatomical differences, which makes the injury more easily detected using radiography. A good lateral screening study is usually sufficient in evaluation of pediatric spine trauma and additional views only increase the radiation dose without increasing sensitivity. Multiple useful lines can be obtained on the lateral cervical radiograph including the anterior cervical line, posterior cervical line, spinolaminar line, Wackenheim clivus line and the $\mathrm{C} 2$ line. The $\mathrm{C} 2$ line is drawn along the posterior $\mathrm{C} 2$ body and should intersect the upper corner of $\mathrm{C} 3$ and is useful for evaluating subluxation/dislocation. The clivus line is drawn along the clivus and should pass though the posterior one-third of the odontoid. The spinolaminar line is most useful in evaluating for pseudosubluxation at C2-3 level. A multitude of measurements are also available, which include the basiondens interval (less than $12 \mathrm{~mm}$ ), Power ratio (less than or equal to 1), and the atlanto-dental distance (less than $5 \mathrm{~mm}$ ). Flexion and extension radiographs have little use in the acute evaluation of pediatric cervical trauma due to patient cooperation, pain and technical limitations.

Multidetector CT (MCDT) is useful in evaluating cervical injury in children, but at a significant radiation cost. This modality should be used if cervical radiographs are unclear or suggest injury or if there is a strong clinical concern. Sagittal and coronal reconstructions are necessary for a complete examination. To reduce radiation dose, limited evaluation of the area of concern might be appropriate and if no fractures are identified imaging of the entire cervical spine might not be warranted. It is important to realize that the measurements used on radiographs are not transferable to the MDCT images. In children, the atlanto-dental distance should be less than 
Fig. 1 Cervical spine examination. a Lateral cervical spine demonstrates slight anterior tilting of the dens. b Sagittal T2-W image with fat suppression shows edema superior and inferior to the $\mathrm{C} 2$ synchondrosis. There is also edema within upper thoracic vertebral bodies consistent with compression injury. MDCT (not shown) demonstrated no widening of the synchondrosis
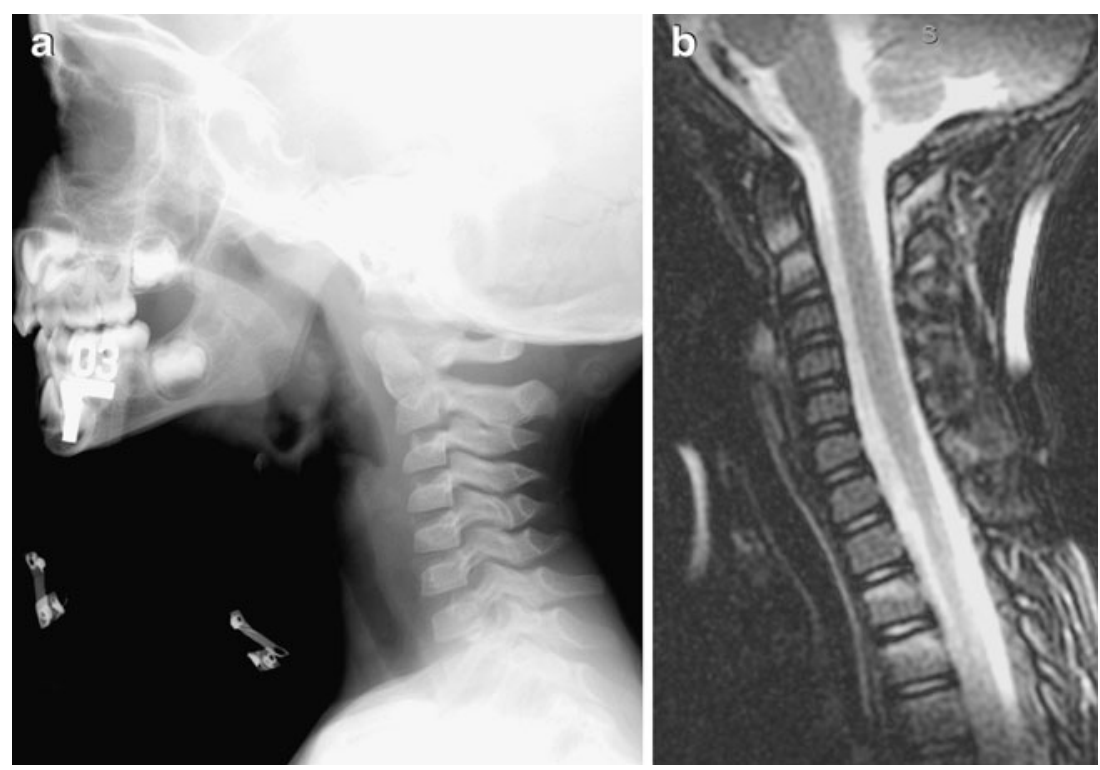

$2.6 \mathrm{~mm}$, the basion dens index is normally less than $8.5 \mathrm{~mm}$ and Power ratio is less than or equal to 0.9. The distance between the occipital condyles and $\mathrm{C} 1$ lateral mass as well as the distance between the lateral masses of $\mathrm{C} 1$ and $\mathrm{C} 2$ should normally be less than $2.5 \mathrm{~mm}$. If this distance is greater than or equal to $2.5 \mathrm{~mm}$, dislocation or dissociation is highly suspicious.

Ligament and soft-tissue evaluation is best done with MRI. Unfortunately, little in the literature distinguishes significant from insignificant findings on MRI and there is evidence that patients with a normal CT and a normal motor exam do not need an MRI. The previously reported 48-h window to evaluate for ligamentous injury is not thought to apply and therefore an expedited MRI is not necessary to see ligamentous injury. MRI is very helpful in clearing a cervical spine that cannot be clinically cleared after $72 \mathrm{~h}$, which includes the obtunded patient. A negative MRI can conclusively exclude cervical spine injury, but the percentage of false-positive scans is unknown at this time. MRI is also helpful in evaluating congenital defects and potential fractures through a synchondrosis, using T2 fat-suppressed imaging (Fig. 1). MRI is useful in the evaluation of patients with neurological symptoms and the possibility of cord injury.

A reasonable approach to the pediatric cervical spine begins with the trauma surgeon, orthopedist and neurosurgeon. A clinical stratification must exist to separate low risk (no imaging) of cervical injury from high risk (imaging). A lateral cervical radiograph should be the initial screening examination with MDCT and MRI representing additional tools in select circumstances. Screening for cervical injury in children with MDCT is not recommended because of the radiation exposure. In the unconscious or obtunded child, MRI has been shown to decrease time to clearance, ICU time and hospital stays. It is uncertain whether the older child would benefit from a more adult-like approach to cervical injury. 